\title{
Sharp bounds on the rate of convergence of the empirical covariance matrix*
}

\author{
Radosław ADAMCZAK ${ }^{\dagger} \quad$ Alexander E. LITVAK Alain PAJOR \\ Nicole TOMCZAK-JAEGERMANN ${ }^{\ddagger}$
}

February 12, 2018

\begin{abstract}
Let $X_{1}, \ldots, X_{N} \in \mathbb{R}^{n}, n \leq N$, be independent centered random vectors with log-concave distribution and with the identity as covariance matrix. We show that with overwhelming probability one has

$$
\sup _{x \in S^{n-1}}\left|\frac{1}{N} \sum_{i=1}^{N}\left(\left|\left\langle X_{i}, x\right\rangle\right|^{2}-\mathbb{E}\left|\left\langle X_{i}, x\right\rangle\right|^{2}\right)\right| \leq C \sqrt{\frac{n}{N}}
$$

where $C$ is an absolute positive constant. This result is valid in a more general framework when the linear forms $\left(\left\langle X_{i}, x\right\rangle\right)_{i \leq N, x \in S^{n-1}}$ and the Euclidean norms $\left(\left|X_{i}\right| / \sqrt{n}\right)_{i \leq N}$ exhibit uniformly a sub-exponential decay. As a consequence, if $A$ denotes the random matrix with columns $\left(X_{i}\right)$, then with overwhelming probability, the extremal singular values $\lambda_{\min }$ and $\lambda_{\max }$ of $A A^{\top}$ satisfy the inequalities $1-C \sqrt{\frac{n}{N}} \leq$ $\frac{\lambda_{\min }}{N} \leq \frac{\lambda_{\max }}{N} \leq 1+C \sqrt{\frac{n}{N}}$ which is a quantitative version of Bai-Yin theorem [4] known for random matrices with i.i.d. entries.
\end{abstract}

Let $X \in \mathbb{R}^{n}$ be a centered random vector whose covariance matrix is the identity and $X_{1}, \ldots, X_{N}$ be independent copies of $X$. Let $A$ be a random $n \times N$ matrix whose columns are $\left(X_{i}\right)$. By $\lambda_{\min }$ (resp. $\lambda_{\max }$ ) we denote the smallest (resp. the largest) singular number of the matrix of empirical covariance $A A^{\top}$. In the study of the local regime in the random matrix theory of particular interest is the limit behavior of extremal values of the spectrum of $A A^{\top}$. In the case of Wishart Ensemble when the coordinates of $X$ are independent, the Bai-Yin theorem [4] establishes the convergence of $\lambda_{\min } / N$ and $\lambda_{\max } / N$ when $n, N \rightarrow \infty$ and $n / N \rightarrow \beta \in(0,1)$, under the assumption of a finite fourth moment. In this note we study the asymptotic non-limit behavior (also called "non-asymptotic" in Statistics) i.e. we look for sharp upper and lower bounds for singular values in terms of $n$ and $N$, when $n \leq N$ are sufficiently large. For example, for Gaussian matrices it is known that singular values satisfy inequalities

$$
1-C \sqrt{\frac{n}{N}} \leq \frac{\lambda_{\min }}{N} \leq \frac{\lambda_{\max }}{N} \leq 1+C \sqrt{\frac{n}{N}}
$$

* The research was conducted while the authors participated in the Thematic Program on Asymptotic Geometric Analysis at the Fields Institute in Toronto in Fall 2010.

${ }^{\dagger}$ Research partially supported by MNiSW Grant no. N N201 397437 and the Foundation for Polish Science.

‡This author holds the Canada Research Chair in Geometric Analysis. 
with probability close to 1 . We obtain the same estimates for large class of random matrices, which in particular do not require that entries of the matrix are independent or that $X_{i}$ 's are identically distributed. Note that the natural question about convergence of singular values in such a case is still open (see 2 for the case of $X_{i}$ having uniform distribution on a rescaled $\ell_{p}^{n}$ ball).

The natural scalar product and Euclidean norm on $\mathbb{R}^{n}$ are denoted by $\langle\cdot, \cdot\rangle$ and $|\cdot|$. We also denote by the same notation $|\cdot|$ the cardinality of a set. By $C, C_{1}, c$ etc. we will denote absolute positive constants.

Let $X_{1}, \ldots, X_{N}$ be a sequence of random vectors in $\mathbb{R}^{n}$ (not necessarily identically distributed). We say that it is uniformly $\psi_{1}$ if for some $\psi>0$,

$$
\sup _{i \leq N} \sup _{y \in S^{n-1}}\left\|\left|\left\langle X_{i}, y\right\rangle\right|\right\|_{\psi_{1}} \leq \psi
$$

where for a random variable $Y \in \mathbb{R},\|Y\|_{\psi_{1}}=\inf \{C>0 ; \mathbb{E} \exp (|Y| / C) \leq 2\}$. We say that it satisfies the boundedness condition with constant $K$ (for some $K \geq 1$ ) if

$$
\mathbb{P}\left(\max _{i \leq N}\left|X_{i}\right| / \sqrt{n}>K \max \left\{1,(N / n)^{1 / 4}\right\}\right) \leq \exp (-\sqrt{n}) .
$$

The main result of this note is the following theorem.

Theorem 1 Let $n \leq N$ be positive integers and $\psi, K \geq 1$. Let $X_{1}, \ldots, X_{N}$ be independent random vectors in $\mathbb{R}^{n}$ satisfying (2) and (3). Then with probability at least $1-2 \exp (-c \sqrt{n})$ one has

$$
\sup _{x \in S^{n-1}}\left|\frac{1}{N} \sum_{i=1}^{N}\left(\left|\left\langle X_{i}, x\right\rangle\right|^{2}-\mathbb{E}\left|\left\langle X_{i}, x\right\rangle\right|^{2}\right)\right| \leq C(\psi+K)^{2} \sqrt{\frac{n}{N}} .
$$

Remarks. 1. Theorem 1 improves estimates obtained in [1 for log-concave isotropic vectors. There, we considered essentially the case of $N$ proportional to $n$, which was sufficient to answer the question of Kannan, Lovász and Simonovits [6], however, for bigger $N$, the results were off by a logarithmic factor. The theorem above removes this factor completely leading to the best possible estimate for an arbitrary $N$, that is to an estimate of the same order as in the Gaussian case.

2. In the case $N<n$ Theorem 2 below together with assumptions (2) and (3) immediately implies that the norm of the matrix $A$ with columns $X_{1}, \ldots, X_{N}$ satisfies

$$
\|A\| \leq C(\psi+K) \sqrt{n}
$$

with probability at least $1-2 \exp (-c \sqrt{n})$. This in turn implies that $\lambda_{\max } \leq C(\psi+K)^{2} n$ and that

$$
\sup _{x \in S^{n-1}}\left|\frac{1}{N} \sum_{i=1}^{N}\left(\left|\left\langle X_{i}, x\right\rangle\right|^{2}-\mathbb{E}\left|\left\langle X_{i}, x\right\rangle\right|^{2}\right)\right| \leq C(\psi+K)^{2} \frac{n}{N}
$$

with the same probability.

As a consequence of Theorem 1 we obtain the following quantitative version of Bai-Yin theorem 4 known for random matrices with i.i.d. entries.

Corollary 1 Let $A$ be a random $n \times N$ matrix, whose columns $X_{1}, \ldots, X_{N}$ are isotropic random vectors satisfying the assumptions of Theorem 1, Then with probability at least $1-2 \exp (-c \sqrt{n})$,

$$
1-C(\psi+K)^{2} \sqrt{\frac{n}{N}} \leq \frac{\lambda_{\min }}{N} \leq \frac{\lambda_{\max }}{N} \leq 1+C(\psi+K)^{2} \sqrt{\frac{n}{N}}
$$


To emphasize the strength of the above results we observe that conditions (2) and (3) are valid for many classes of distributions.

Example 1 Random vectors uniformly distributed on the Euclidean ball of radius $K \sqrt{n}$ clearly satisfy (3). They also satisfy (2) with $\psi=C K$.

Example 2 Log-concave isotropic random vectors in $\mathbb{R}^{n}$. Recall that a random vector is isotropic if its covariance matrix is the identity and it is log-concave if its distribution has a log-concave density. Such vectors satisfy (2) and (31) for appropriate absolute constants $\psi$ and $K$. The boundedness condition follows from Paouris' theorem ([7) and is explicitly written e.g., in 1], Lemma 3.1. We would like to remark that a version of Theorem 1 with a weaker probability estimate was proved by Aubrun in the case of isotropic log-concave random vectors under an additional assumption of unconditionality (see [3]).

Example 3 Any isotropic random vectors $\left(X_{i}\right)_{i \leq N}$ in $\mathbb{R}^{n}$, satisfying the Poincaré inequality with constant $L$, i.e. such that $\left.\operatorname{Var}\left(f\left(X_{i}\right)\right) \leq L^{2} \mathbb{E} \mid \nabla f\left(X_{i}\right)\right)\left.\right|^{2}$ for all compactly supported smooth functions, satisfy (2) with $\psi=C L$ and (3) with $K=C L$. The question from [5] whether all log-concave isotropic random vectors satisfy the Poincaré inequality with an absolute constant is one of the major open problems in the theory of log-concave measures.

The proof of Theorem 1 is close to arguments in Section 4.3 of [1, however it uses a choice of parameters more appropriate for the case considered here, and a new approximation argument. We need additional notations. Let $1 \leq m \leq N$. By $U_{m}$ we denote the subset of all vectors in $S^{N-1}$ having at most $m$ non-zero coordinates. For an $n \times N$ matrix $A$ we let

$$
A_{m}=\sup _{z \in U_{m}}|A z| .
$$

The main technical tool is the following result which is the "in particular" part of Theorem 3.13 from [1] in which one needs to adjust corresponding constants and to take a union bound.

Theorem 2 Let $X_{1}, \ldots, X_{N}$ be as in Theorem 1 , let $A$ be a random $n \times N$ matrix whose columns are the $X_{i}$ 's. Then for every $t \geq 1$ one has

$$
\mathbb{P}\left(\exists m \quad A_{m} \geq C \psi t \max \left\{\sqrt{m} \ln \frac{2 N}{m}, \sqrt{n}\right\}+6 \max _{i \leq N}\left|X_{i}\right|\right) \leq \exp (-t \sqrt{n}) .
$$

Proof of Theorem 1, For $x \in S^{n-1}$ set

$$
S(x)=\left|\frac{1}{N} \sum_{i=1}^{N}\left(\left|\left\langle X_{i}, x\right\rangle\right|^{2}-\mathbb{E}\left|\left\langle X_{i}, x\right\rangle\right|^{2}\right)\right| .
$$

Let $B>0$ be a parameter which we specify later and observe that

$$
\begin{aligned}
& \sup _{x \in S^{n-1}} S(x) \leq \sup _{x \in S^{n-1}}\left(\left|\frac{1}{N} \sum_{i=1}^{N}\left(\left(\left|\left\langle X_{i}, x\right\rangle\right| \wedge B\right)^{2}-\mathbb{E}\left(\left|\left\langle X_{i}, x\right\rangle\right| \wedge B\right)^{2}\right)\right|\right. \\
& \left.+\frac{1}{N} \sum_{i=1}^{N}\left(\left|\left\langle X_{i}, x\right\rangle\right|^{2}-B^{2}\right) \mathbf{1}_{\left\{\left|\left\langle X_{i}, x\right\rangle\right| \geq B\right\}}+\frac{1}{N} \mathbb{E} \sum_{i=1}^{N}\left(\left|\left\langle X_{i}, x\right\rangle\right|^{2}-B^{2}\right) \mathbf{1}_{\left\{\left|\left\langle X_{i}, x\right\rangle\right| \geq B\right\}}\right) .
\end{aligned}
$$


We denote the summands under the supremum by $S_{1}(x), S_{2}(x)$, and $S_{3}(x)$, respectively.

Estimate for $S_{1}$ : Given $x \in S^{n-1}$ and $i \leq N$ let $Z_{i}=Z_{i}(x)=\left(\left|\left\langle X_{i}, x\right\rangle\right| \wedge B\right)^{2}-\mathbb{E}\left(\left|\left\langle X_{i}, x\right\rangle\right| \wedge B\right)^{2}$. Then $\left|Z_{i}\right| \leq B^{2}$. Moreover, since

$$
\operatorname{Var}\left(Z_{i}\right) \leq \mathbb{E}\left(\left|\left\langle X_{i}, x\right\rangle\right| \wedge B\right)^{4} \leq \mathbb{E}\left|\left\langle X_{i}, x\right\rangle\right|^{4} \leq C_{1} \psi^{4},
$$

we observe that $\sigma^{2}=\frac{1}{N} \sum_{i=1}^{N} \operatorname{Var}\left(Z_{i}\right) \leq C_{1} \psi^{4}$. Thus, by Bernstein's inequality

$$
\mathbb{P}\left(S_{1}(x) \geq \theta\right)=\mathbb{P}\left(\frac{1}{N} \sum_{i=1}^{N} Z_{i} \geq \theta\right) \leq \exp \left(-\frac{\theta^{2} N}{2\left(C_{1} \psi^{4}+B^{2} \theta / 3\right)}\right) .
$$

It is well known that $S^{n-1}$ admits a $(1 / 3)$-net $\mathcal{N}$ in the Euclidean metric such that $|\mathcal{N}| \leq 7^{n}$. Then by the union bound we obtain that if

$$
\theta^{2} N>8 C_{1} \psi^{4} n \ln 7 \quad \text { and } \quad \theta N>(8 / 3) B^{2} n \ln 7
$$

then

$$
\mathbb{P}\left(\sup _{x \in \mathcal{N}} S_{1}(x) \geq \theta\right) \leq \exp \left(n \ln 7-\frac{\theta^{2} N}{2\left(C_{1} \psi^{4}+B^{2} \theta / 3\right)}\right) \leq \exp \left(-\frac{\theta^{2} N}{4\left(C_{1} \psi^{4}+B^{2} \theta / 3\right)}\right) .
$$

Estimates for $S_{2}$ and $S_{3}$ : By Hölder's inequality and (2) we have, for some absolute constant $C_{2} \geq 1$,

$$
\sup _{x \in S^{n-1}} S_{3}(x) \leq \frac{1}{N} \sum_{i=1}^{N} \sup _{x \in S^{n-1}}\left\|\left\langle X_{i}, x\right\rangle\right\|_{4}^{2} \mathbb{P}\left(\left|\left\langle X_{i}, x\right\rangle\right| \geq B\right)^{1 / 2} \leq C_{2} \psi^{2} \exp (-B / \psi) .
$$

To estimate $S_{2}$, we will use the following notation

$$
M=\max \left\{\psi^{2} n, \max _{i \leq N}\left|X_{i}\right|^{2}\right\}, \quad E_{B}=E_{B}(x)=\left\{i \leq N:\left|\left\langle X_{i}, x\right\rangle\right| \geq B\right\}, \quad m=\sup _{x \in S^{n-1}}\left|E_{B}(x)\right| .
$$

By the definition of $A_{m}$, we have for every $x \in S^{n-1}$

$$
B^{2}\left|E_{B}\right| \leq \sum_{i \in E_{B}}\left|\left\langle X_{i}, x\right\rangle\right|^{2} \leq \sup _{|E| \leq m} \sum_{i \in E}\left|\left\langle X_{i}, x\right\rangle\right|^{2} \leq A_{m}^{2}
$$

which yields $B^{2} m \leq A_{m}^{2}$ and $N S_{2}(x) \leq A_{m}^{2}$. Theorem 2 implies that for some absolute constant $C \geq C_{2}$, with probability at least $1-\exp (-\sqrt{n})$ one has

$$
B^{2} m \leq C\left(M+\psi^{2} m \ln ^{2} \frac{2 N}{m}\right) \text { and } \sup _{x \in S^{n-1}} S_{2}(x) \leq C\left(\frac{M}{N}+\psi^{2} \frac{m}{N} \ln ^{2} \frac{2 N}{m}\right) .
$$

Now we choose the parameters. Let $B=2 \sqrt{2 C} \psi \ln (5 N / n)$. Then (77) gives $S_{3}(x) \leq C \psi^{2} \frac{n}{N} \leq C \frac{M}{N}$ for all $x \in S^{n-1}$ and together with (8) it yields that with probability at least $1-\exp (-\sqrt{n})$ one has

$$
\sup _{x \in S^{n-1}}\left(S_{2}(x)+S_{3}(x)\right) \leq C\left((2 M / N)+\psi^{2}(m / N) \ln ^{2}(2 N / m)\right) .
$$

It is easy to check that $M \geq \psi^{2} m \ln ^{2}(2 N / m)$ on the set where (8) holds. Indeed, assume it is not so, thus $M<\psi^{2} m \ln ^{2}(2 N / m)$. Then by (8) we observe that $B^{2} \leq 2 C \psi^{2} \ln ^{2}(2 N / m)$, which implies

$$
m \leq 2 N \exp (-B / \psi \sqrt{2 C})=2 n^{2} / 25 N .
$$


By our hypothetical upper bound for $M$ and since $f(m)=m \ln ^{2}(2 N / m)$ increases on $\left[1,2 N / e^{2}\right]$, we get

$$
\psi^{2} n \leq M \leq \psi^{2}\left(8 n^{2} / 25 N\right) \ln ^{2}(5 N / n),
$$

which is impossible.

It follows that

$$
\mathbb{P}\left(\sup _{x \in S^{n-1}}\left(S_{2}(x)+S_{3}(x)\right) \leq 3 C(M / N)\right) \geq 1-\exp (-\sqrt{n}) .
$$

Combining this estimate with (무), we get

$$
\mathbb{P}\left(\sup _{x \in \mathcal{N}} S(x) \leq \theta+3 C \frac{M}{N}\right) \geq 1-\exp (-\sqrt{n})-\exp \left(-\frac{\theta^{2} N}{4\left(C_{1} \psi^{4}+B^{2} \theta / 3\right)}\right) .
$$

We now set $\theta=C_{3} \psi^{2} \sqrt{n / N}$, where $C_{3}$ is a sufficiently large absolute positive constant so that (5) is satisfied. Then using boundedness condition with constant $K$ we obtain

$$
\mathbb{P}\left(\sup _{x \in \mathcal{N}} S(x) \leq\left(C_{3} \psi^{2}+3 C K^{2}\right) \sqrt{n / N}\right) \geq 1-\exp (-\sqrt{n})-\exp (-c n) \geq 1-2 \exp (-c \sqrt{n}),
$$

where $c$ is a sufficiently small positive constant. It proves the desired estimate on the $(1 / 3)$-net.

To pass from $\mathcal{N}$ to the whole sphere note that $S(x)$ can be written as $|\langle T x, x\rangle|$, where $T$ is a self-adjoint operator on $\mathbb{R}^{n}$. Thus, writing for each $x \in S^{n-1}, x=y+z$ with $y \in \mathcal{N}$ and $|z| \leq 1 / 3$, we get

$$
\|T\|=\sup _{x \in S^{n-1}}|\langle T x, x\rangle| \leq \sup _{y \in \mathcal{N}}|\langle T y, y\rangle|+\frac{2}{3} \sup _{y \in \mathcal{N}}|T y|+\sup _{|z| \leq 1 / 3}|\langle T z, z\rangle| \leq \sup _{y \in \mathcal{N}} S(y)+\frac{7}{9}\|T\|,
$$

which implies the desired estimate on the whole sphere $S^{n-1}$.

\section{References}

[1] R. Adamczak, A. E. Litvak, A. Pajor and N. Tomczak-Jaegermann, Quantitative estimates of the convergence of the empirical covariance matrix in log-concave Ensembles, Journal of AMS, 234 (2010), $535-561$.

[2] G. Aubrun, Random points in the unit ball of $\ell_{p}^{n}$, Positivity, 10 (2006), 755-759.

[3] G. Aubrun, Sampling convex bodies: a random matrix approach, Proc. AMS, 135 (2007), 1293-1303.

[4] Z. D. Bai and Y. Q. Yin, Limit of the smallest eigenvalue of a large dimensional sample covariance matrix, Ann. Probab. 21 (1993), 1275-1294.

[5] R. Kannan, L. Lovász and M. Simonovits, Isoperimetric problems for convex bodies and a localization lemma. Discrete Comput. Geom. 13 (1995), no. 3-4, 541-559.

[6] R. Kannan, L. Lovász and M. Simonovits, Random walks and $O^{*}\left(n^{5}\right)$ volume algorithm for convex bodies, Random structures and algorithms, 2 (1997), no. 1, 1-50.

[7] G. Paouris, Concentration of mass on convex bodies. Geom. Funct. Anal. 16 (2006), no. 5, 1021-1049. 
Radosław Adamczak

Institute of Mathematics,

University of Warsaw

Banacha 2, 02-097 Warszawa, Poland

R.Adamczak@mimuw.edu.pl

Alexander E. Litvak

Department of Mathematical and Statistical Sciences,

University of Alberta,

Edmonton, Alberta, Canada T6G 2G1

alexandr@math . ualberta.ca

Alain Pajor

Equipe d'Analyse et Mathématiques Appliquées,

Université Paris Est,

5 boulevard Descartes, Champs sur Marne, 77454 Marne-la-Vallee,

Cedex 2, France

alain.pajor@univ-mlv.fr

Nicole Tomczak-Jaegermann,

Department of Mathematical and Statistical Sciences,

University of Alberta,

Edmonton, Alberta, Canada T6G 2G1

nicole@ellpspace.math.ualberta.ca 